\title{
Correction to: A modeling study of spinal motoneuron recruitment regulated by ionic channels during fictive locomotion
}

\author{
Qiang Zhang ${ }^{1} \cdot$ Yue Dai ${ }^{1,2}$
}

Published online: 28 January 2021

○) Springer Science+Business Media, LLC, part of Springer Nature 2020

\section{Correction to: Journal of Computational Neuroscience https://doi.org/10.1007/s10827-020-00763-4}

The authors find several printing errors in the equations in the final versions on line and in print proof. However, there were no such errors in the submitted proof.

The below equation should be corrected from

$$
\begin{gathered}
N a T: I_{N a T}=g_{N a T} \cdot m^{3} \cdot h \cdot\left(V_{m} \cdot E_{N a}\right) \\
N a T: I_{N a P}=g_{N a P} \cdot m \cdot\left(V_{m} \cdot E_{N a}\right) \\
K(D R): I_{K(D R)}=g_{K(D R)} \cdot n^{4} \cdot\left(V_{m} \cdot E_{K}\right) \\
K(A H P):\left\{\begin{array}{c}
I_{K(A H P)}=g_{K(A H P)} \cdot g \cdot\left(V_{m}-E_{K}\right) \\
I_{C a L}=g_{C a L} \cdot m_{C a L} \cdot\left(V_{m}-E_{C a}\right)
\end{array}\right. \\
H: I_{H}=g_{H} \cdot m_{H} \cdot\left(V_{m} \cdot E_{H}\right) \\
L_{-} C a_{L}: I_{L \_C a_{L}}=g_{L \_C a_{L}} \cdot m_{L \_C a_{L}} \cdot\left(V_{m}-E_{C a}\right) \\
H_{-} C a_{L}: I_{H \_C a_{L}}=g_{H_{-} C a_{L}} \cdot m_{H \_C a_{L}} \cdot\left(V_{m} \cdot E_{C a}\right) \\
\text { Leak }: I_{\text {Leak }}=g_{\text {Leak }} \cdot\left(V_{m}-E_{\text {Leak }}\right)
\end{gathered}
$$

To

$$
\begin{aligned}
& N a T: I_{N a T}=g_{N a T} \cdot m^{3} \cdot h \cdot\left(V_{m}-E_{N a}\right) \\
& N a P: I_{N a P}=g_{N a P} \cdot m \cdot\left(V_{m}-E_{N a}\right) \\
& K(D R): I_{K(D R)}=g_{K(D R)} \cdot n^{4} \cdot\left(V_{m}-E_{K}\right) \\
& K(A H P):\left\{\begin{array}{l}
I_{K(A H P)}=g_{K(A H P)} \cdot q \cdot\left(V_{m}-E_{K}\right) \\
I_{C a L}=g_{C a L} \cdot m_{C a L} \cdot\left(V_{m}-E_{C a}\right)
\end{array}\right. \\
& H: I_{H}=g_{H} \cdot m_{H} \cdot\left(V_{m}-E_{H}\right) \\
& L_{C} C a_{L}: I_{L_{-} C a_{L}}=g_{L_{-} C a_{L}} \cdot m_{L_{-} C a_{L}} \cdot\left(V_{m}-E_{C a}\right) \\
& H_{C} C a_{L}: I_{H_{-} C a_{L}}=g_{H_{-} C a_{L}} \cdot m_{H_{-} C a_{L}} \cdot\left(V_{m}-E_{C a}\right) \\
& \text { Leak }: I_{\text {Leak }}=g_{\text {Leak }}\left(V_{m}-E_{\text {Leak }}\right)
\end{aligned}
$$

The original article has been corrected.

The original article can be found online at https://doi.org/10.1007/ s10827-020-00763-4.

Yue Dai

ydai@tyxx.ecnu.edu.cn

1 Shanghai Key Laboratory of Multidimensional Information Processing, School of Communication and Electronic Engineering, East China Normal University, Shanghai 200241, China

2 Key Laboratory of Adolescent Health Assessment and Exercise Intervention of Ministry of Education, School of Physical Education and Health Care, East China Normal University, Shanghai 200241, China 